\title{
Avaliação do eixo hipotálamo- hipófise adrenal no diagnóstico e na remissão da doença de Cushing
}

\author{
Assessment of the hypothalamic-pituitary-adrenal axis \\ in Cushing's disease diagnosis and remission
}

Fabíola Costenaro', Ticiana C. Rodrigues ${ }^{1,2}$, Guilherme A. F. Rollin',

Mauro A. Czepielewski ${ }^{1,2}$

\section{SUMÁRIO}

A doença de Cushing (DC) permanece um desafio médico com muitas questões ainda não respondidas. $O$ sucesso terapêutico dos pacientes com DC está ligado à correta investigação do diagnóstico síndrômico e etiológico, além da experiência e talento do neurocirurgião. A adenomectomia hipofisária transesfenoidal constitui-se no tratamento de escolha para a DC. A avaliação da remissão da doença no pós-operatório e da recorrência em longo prazo constitui um desafio ainda maior. Especial destaque deve ser dado para o cortisol sérico no pós-operatório como marcador de remissão. Adicionalmente, o uso de corticoide exógeno no pós-operatório apenas em vigência de insuficiência adrenal tem sido sugerido por alguns autores como requisito essencial para permitir a correta interpretação do cortisol sérico nesse cenário. Neste artigo, revisamos as formas de avaliação da atividade da DC e os marcadores de remissão e recidiva da DC após a realização da cirurgia transesfenoidal. Arq Bras Endocrinol Metab. 2012;56(3):159-67

Descritores

Doença de Cushing; cortisol sérico; remissão; cirurgia hipofisária

\section{SUMMARY}

Cushing's disease $(C D)$ remains a medical challenge, with many questions still unanswered. Successful treatment of CD patients is closely related to correct approach to syndromic and etiological diagnosis, besides the experience and talent of the neurosurgeon. Pituitary transsphenoidal adenomectomy is the treatment of choice for DC. Assessment of remission after surgery and recurrence in the long term is an even greater challenge. In this regard, special attention should be paid to the role of postoperative serum cortisol as a marker of CD remission. Additionally, the postoperative use of exogenous glucocorticoids only in cases of adrenal insufficiency has been suggested by some authors as an essential practice to enable the use of serum cortisol in this scenario. In this article, we review the forms of evaluation of DC activity, and markers of remission and relapse of CD after transsphenoidal surgery. Arq Bras Endocrinol Metab. 2012;56(3):159-67

\section{Keywords}

Cushing's disease; serum cortisol; remission; pituitary surgery

\section{INTRODUÇÃO}

\footnotetext{
doença de Cushing (DC) apresenta incidência Lestimada de 0,7 a 3 casos/milhão/ano (1-3), com prevalência de 39,1 casos por milhão de habitantes (4). Acomete adultos jovens com pico de incidência entre a terceira e quarta décadas de vida (5) e predominantemente mulheres.
}

1 Programa de Pós-Graduação em Ciências Médicas -

Endocrinologia, Universidade Federal do Rio Grande do Sul (UFRGS), Porto Alegre, RS, Brasil ${ }^{2}$ Serviço de Endocrinologia, Hospital de Clínicas de Porto Alegre, Porto Alegre, RS, Brasil

\author{
Correspondência para: \\ Mauro A. Czepielewski \\ Serviço de Endocrinologia, \\ Hospital de Clínicas de Porto Alegre \\ Rua Ramiro Barcellos, 2350 \\ prédio $12,4 \circ$ andar \\ 90035-003 - Porto Alegre, RS, Brasil \\ maurocze@terra.com.br
}

Recebido em 1/Dez/2011 Aceito em 14/Fev/2012
Para o sucesso no manejo desses pacientes, é necessário que a investigação diagnóstica obedeça a uma sequência lógica: inicialmente o diagnóstico sindrômico e posteriormente o diagnóstico etiológico.

$\mathrm{O}$ risco relativo de morbidade e mortalidade na DC é de 2 a 5 vezes acima da população em geral $(2,3)$, podendo equiparar-se ao risco da população em geral durante a remissão da doença (2). 
Em decorrência da relativa raridade da DC, poucos centros médicos têm experiência suficiente para estabelecer protocolos diagnósticos e terapêuticos baseados em suas casuísticas. Este artigo de revisão tem por objetivo auxiliar o endocrinologista clínico na tomada de decisões sobre a DC. Revisamos as formas de avaliação da atividade da doença e os marcadores de remissão e recidiva após a realização da cirurgia transesfenoidal.

\section{SÍNDROME DE CUSHING}

O diagnóstico da síndrome de Cushing (SC) é realizado por meio da presença de manifestações clínicas sugestivas de hipercortisolismo associadas a exames laboratoriais confirmatórios da hipersecreção de cortisol.

As manifestações clínicas mais específicas da SC incluem: pletora e rubicundez facial, fragilidade capilar e estrias violáceas na pele, fraqueza muscular proximal, osteopenia/osteoporose e ganho ponderal. Em crianças, a característica típica é o ganho de peso com redução da velocidade de crescimento.

Entre as manifestações menos específicas, estão a obesidade centrípeta, a hipertensão arterial sistêmica, os diferentes estágios de hiperglicemia incluindo o diabetes, os transtornos psiquiátricos e o hipogonadismo/irregularidade menstrual $(1,3)$. Aproximadamente $20 \%$ a $47 \%$ dos pacientes com SC possuem intolerância à glicose $\mathrm{e} / \mathrm{ou}$ diabetes e mais de $70 \%$ deles são hipertensos (5). Considerando-se que a prevalência de diabetes melito na população brasileira em geral é de 9,7\% [Intervalo de confiança (IC) 95\%: 9,0\%-10,3\%] (6), e a prevalência de hipertensão arterial em adultos brasileiros é de 23,9\% (IC 95\%: 23\%-24,7\%) (7), podemos perceber a baixa especificidade desses caracteres clínicos para a presença da SC.

Uma situação clínica que deve sempre exigir atenção médica é a utilização de glicocorticoides exóge- nos, que podem corresponder a $99 \%$ dos casos de SC (8). Uma vez que essa situação tenha sido descartada, o hipercortisolismo endógeno poderá ser investigado.

Entre os exames confirmatórios do hipercortisolismo, estão a ausência de supressão do cortisol após $1 \mathrm{mg}$ de dexametasona "overnight" $(1,3,9,10)$, o excesso de excreção do cortisol livre urinário de $24 \mathrm{~h}$ (CLU) $(1,3,11)$ e a presença de um cortisol elevado à meia-noite, seja por cortisol salivar $(3,12)$ ou por cortisol sérico - este último pode ser coletado com o indivíduo dormindo $(1,3,13)$ ou acordado $(1,3,14)$-, e ainda o cortisol sérico não suprimido após $2 \mathrm{mg} / \mathrm{dia}$ de dexametasona em $48 \mathrm{~h}(0,5 \mathrm{mg} 6 / 6 \mathrm{~h}$ - teste de Liddle I $)$ $(1,3)$ (Tabela 1).

Importante ressaltar que é necessária a presença de dois ou mais testes alterados para realização do diagnóstico sindrômico $(1,3)$. Com a validação do cortisol salivar à meia-noite, tornou-se possível a avaliação do ritmo circadiano nos pacientes ambulatoriais, tendo em vista que o cortisol plasmático à meia-noite necessita de internação hospitalar para sua realização. A excelente acurácia da maioria dos testes utilizados no diagnóstico sindrômico (Tabela 1) foi estabelecida em pacientes com doença moderada à grave.

Recentemente diagnósticos mais precoces estão sendo realizados na prática clínica, muitas vezes com apresentação leve da doença. Nesse sentido, para a exclusão do diagnóstico da SC, é necessária a realização de, no mínimo, dois testes em momentos diferentes $(1,15)$, tendo em vista a elevada prevalência de um dos testes normais nos casos de SC leve ou cíclica: 54\% para cortisol após $1 \mathrm{mg}$ de dexametasona “overnight" e $92 \%$ para cortisol salivar (15).

Uma vez diagnosticada a SC, é necessário estabelecer seu diagnóstico etiológico. A SC endógena origina-se de um corticotrofinoma hipofisário em $70 \%-80 \%$ dos casos $(1,3)$, caracterizando a doença de Cushing (DC).

Tabela 1. Performance dos testes no diagnóstico da síndrome de Cushing

\begin{tabular}{lccc}
\hline \multicolumn{1}{c}{ Teste } & Ponto de corte & Sensibilidade (\%) & Especificidade (\%) \\
\hline Cortisol após 1 mg de dexametasona "overnight" $(3,9,10)$ & $\geq 1,8 \mu \mathrm{g} / \mathrm{dL}$ & 95 & 80 \\
& $\geq 5 \mu \mathrm{g} / \mathrm{dL}$ & 85 & 95 \\
Cortisolúria de 24h (3,11) & $>$ Limite superior da normalidade & $90-100$ & $50-98$ \\
Cortisol salivar à meia-noite (12) & $>145 \mathrm{ng} / \mathrm{dL}$ & $95-100$ & $93-100$ \\
Cortisol sérico à meia-noite (13,14) & Dormindo: $>1,8 \mu \mathrm{g} / \mathrm{dL}$ & 100 & 20 \\
& Acordado: $>7,5 \mu \mathrm{g} / \mathrm{dL}$ & 96 & $83-96$ \\
Teste de Liddle I (1,3) & $>1,8-5 \mu \mathrm{g} / \mathrm{dL}$ & $67-95$ & $70-100$ \\
\hline
\end{tabular}

CRH: hormônio liberador de cortrosina; DDAVP: desmopressina. 


\section{DOENÇA DE CUSHING}

A DC caracteriza-se em $90 \%$ dos casos por microadenoma hipofisário. Em razão da elevada morbimortalidade da doença ativa, é fundamental o diagnóstico preciso para obter-se tratamento eficaz, com resultados rápidos e remissão em longo prazo.

O diagnóstico etiológico de DC nos pacientes com SC é estabelecido por meio de: 1) ACTH sérico normal ou elevado; 2) supressão do cortisol sérico ou urinário após a administração de $8 \mathrm{mg}$ de dexametasona "overnight" ou $2 \mathrm{mg} \mathrm{6/6h}$ por $48 \mathrm{~h}$ (Liddle II) $(3,16) ; 3)$ presença de adenoma em imagem hipofisária por tomografia computadorizada (TC) [Sensibilidade (S): 20\%-50\%] ou por ressonância magnética $(\mathrm{RM})(\mathrm{S}: 60 \%-70 \%)(9,17)$. Devido à baixa sensibilidade da TC de sela túrcica, sugere-se sempre como método de imagem de escolha a utilização da RM de sela túrcica para a investigação da DC; 4) nos pacientes com imagem hipofisária negativa, é necessária a presença de gradiente do ACTH durante cateterismo bilateral simultâneo do seio petroso inferior com CRH ou com desmopressina (DDAVP) $(3,18)$; $5)$ adicionalmente, pode ser utilizado o estímulo periférico do cortisol/ACTH ao CRH seguido ou não da supressão de dexametasona pelo Liddle II $(1,19,20)$ ou do ACTH e/ou cortisol ao DDAVP como ferramenta auxiliar no diagnóstico da DC (Tabela 2).

Em relação ao teste com estímulo ao $\mathrm{CRH}$, a resposta do ACTH periférico ao $\mathrm{CRH}>50 \%$ no diagnóstico diferencial da DC dos tumores ectópicos produtores de ACTH apresenta uma acurácia diagnóstica de 86\% (9) (Tabela 2). A utilização do $\mathrm{CRH}$ no diagnóstico diferencial do pseudo-Cushing (PC) e DC tem uma S: $98 \%-100 \%$ e E: $60 \%-62,5 \%$ para a elevação do cortisol sérico maior de $1,4 \mu \mathrm{g} / \mathrm{dL}$ com o estímulo do CRH ovino aplicado na manhã seguinte após o término do teste de Liddle II $(1,19)$.

Recentemente, Arnaldi e cols. (20) analisaram o valor basal do cortisol e do ACTH basal para des- cartar PC e concluíram que a associação dos valores basais do cortisol > $12 \mu \mathrm{g} / \mathrm{dL}$ e do ACTH > $54 \mathrm{pg} /$ $\mathrm{mL}$ apresentou S: 91\% e E: 98\%. Quando avaliados os picos hormonais, observou-se que a associação do pico de cortisol $>21 \mu \mathrm{g} / \mathrm{dL}$ e do ACTH $>45 \mathrm{pg} /$ $\mathrm{mL}$ após $\mathrm{CRH}$ humano apresentou uma S: $95 \% \mathrm{e}$ uma E: 91\% para o diagnóstico diferencial de DC e PC. Além disso, a elevação do cortisol acima de $14 \%$ em relação ao cortisol basal excluiu $100 \%$ dos pacientes com tumor ectópico produtor de ACTH (20). Entretanto, apesar dos bons resultados com a utilização do CRH, ele apresenta o inconveniente de ser de difícil importação para o Brasil e possuir um alto custo.

O DDAVP é um análogo sintético da vasopressina, sem efeito vasopressor, que atua especificamente nos receptores V2 renais e nos receptores V3 que estão "up-regulated” nos adenomas corticotróficos (21). Dessa maneira, induz uma significativa elevação do cortisol e do ACTH na maioria dos pacientes com DC. Com relação ao teste do DDAVP periférico, deve ser levado em conta o fato de que alguns tumores produtores de ACTH ectópico, especialmente o carcinoide brônquico, respondem ao DDAVP (1), prejudicando a acurácia desse teste na diferenciação da DC dos tumores ectópicos produtores de ACTH. Por outro lado, esse teste tem se mostrado útil na diferenciação da DC dos casos de PC (mais comumente depressão, alcoolismo e obesidade mórbida). Nesse cenário, Moro e cols. (22) e Pecori Giraldi e cols. (19) observaram acurácia de $85 \%$-94\% para o pico de ACTH após DDAVP (Tabela 2). Tirabassi e cols. (23) também encontraram boa acurácia (90\%) para o incremento do ACTH > 18 $\mathrm{pg} / \mathrm{dL}$ após DDAVP associado ao cortisol sérico basal $>12 \mathrm{ng} / \mathrm{dL}$ na diferenciação da DC com estados de PC. Em razão da relativa raridade da DC, poucos centros médicos têm experiência suficiente para estabelecer protocolos diagnósticos e terapêuticos baseados em suas casuísticas.

Tabela 2. Performance dos testes para diagnóstico etiológico da doença de Cushing

\begin{tabular}{lccc}
\hline Teste & Ponto de corte & Sensibilidade (\%) & Especificidade (\%) \\
\hline Liddle II (3,16) & Cortisol sérico ou urinário < 50\% basal & $65-100$ & $50-100$ \\
Cateterismo bilateral simultâneo do seio petroso inferior & Gradiente central/periferia ACTH $>2$ & & 96 \\
$(3,18)$ & basal ou $>3$ após CRH & $84-95$ & $62-100$ \\
& $>3$ após DDAVP & 85 & 100 \\
Estímulo ao CRH periférico (9) & ACTH $>50 \%$ do basal & $82-87$ & $90-91$ \\
Estímulo ao DDAVP periférico $(19,22)^{*}$ & Pico ACTH $>27$ pg/dL do basal & \\
\hline
\end{tabular}

* No diagnóstico diferencial com pseudo-Cushing. 


\section{TERAPÊUTICA DA DOENÇA DE CUSHING}

Atualmente, a cirurgia hipofisária transesfenoidal (CTE) com adenomectomia seletiva constitui-se no tratamento de escolha para a maioria dos pacientes com DC (24).

Há evidências de que pacientes com DC curados após CTE apresentem mortalidade em longo prazo semelhante à da população em geral, controlada para sexo e idade $(2,3)$. A taxa de remissão após CTE observada em bons centros varia entre 53\%-96\% (9,2528 ), chegando a $96 \%$ nos microadenomas (26) e tão baixas quanto $53 \%$ nos macroadenomas (27). Além disso, as taxas de cura na segunda CTE são inferiores às da primeira CTE e variam em torno de 28\%-67\% (9,28-31), já as chances de hipopituitarismo aumentam nas reintervenções, alcançando taxas de 46\%100\% após a segunda CTE (28-31).

A possibilidade de reintervenção cirúrgica precoce (menos de 15 dias de intervalo entre as CTE), objetivando-se encontrar menor reação local cicatricial com menor mudança da anatomia da sela, foi proposta por alguns autores $(31,32)$. Na casuística de Locatelli e cols. (31), apesar do sucesso em 67\% dos 12 casos, $100 \%$ dos indivíduos desenvolveram algum grau de hipopituitarismo após a segunda intervenção cirúrgica e, em $83 \%$ dos casos, houve alguma complicação leve no pós-operatório. Na publicação de Rollin e cols. (28), a taxa de remissão foi de $0 \%$ nos cinco pacientes com doença persistente submetidos precocemente à segunda CTE.

A recidiva da DC ocorre em $5 \%$ a $64 \%$ das vezes em até cinco anos após a CTE $(9,28,30,33)$. Essas discrepâncias entre os diferentes centros devem-se aos diferentes critérios de remissão adotados, à quantidade de reintervenções cirúrgicas e à duração do seguimento dos pacientes.

\section{REMISSÃO E RECORRÊNCIA DA DOENÇA DE CUSHING APÓS A CIRURGIA HIPOFISÁRIA}

Pacientes sem remissão da DC pela CTE ou com risco de recidiva da doença precisam ser identificados precocemente para que se possa introduzir um segundo tratamento que seja efetivo assim que a falência terapêutica tenha sido diagnosticada. Nesse cenário, critérios de remissão fidedignos são essenciais para a definição de remissão da DC, e a identificação de marcadores capazes de predizer a recidiva da doença é fundamental para o seguimento desses pacientes (34).

Os critérios mais aceitos para remissão da DC são a resolução clínica do hipercortisolismo associada a algum dos seguintes critérios: cortisol suprimido após a administração de $1 \mathrm{mg}$ de dexametasona overnight (35-37), dois ou três CLU dentro do limite da normalidade $(9,34,38,39)$, dependência ao uso de glicocorticoide exógeno por insuficiência adrenal $(29,32)$ ou mesmo algum desses critérios isolados ou em associação. No entanto, a melhor maneira de avaliar a remissão e o risco de recidiva da DC no PO ainda não está definida.

A avaliação das chances de remissão da DC inicia-se antes mesmo da realização da CTE, pois pacientes com doença mais severa, com tumores maiores (macroadenomas) e com extensão além da sela túrcica, geralmente possuem menor chance de remissão da $\operatorname{DC}(26,29,34,36)$. Apesar disso, um grupo não identificou a influência do tamanho tumoral, invasão tumoral ou crescimento extrasselar como marcadores de desfechos cirúrgicos (25). Os pacientes com presença de lesão bem visualizada no exame de imagem hipofisária (TC ou RM) são considerados como tendo maiores chances de remissão $(25,40)$. A experiência e a habilidade do neurocirurgião também são fatores de grande impacto para o sucesso da cirurgia $(41,42)$.

A presença de um adenoma produtor de ACTH no anatomopatológico do PO também é considerada um dos fatores associados à maior remissão da DC $(25,27,38)$. Entretanto, Chee e cols. (30) observaram que o anatomopatológico com presença de adenoma foi um bom marcador de remissão da DC apenas quando em associação ao achado radiológico positivo. O tempo de utilização de glicocorticoide pelos pacientes no PO também é sugerido como inversamente relacionado ao risco de recorrência da DC $(25,28)$. Em contrapartida, Blevins e cols. (29) não encontraram correlação entre a duração da insuficiência adrenal com tempo de remissão da DC nos pacientes com macroadenomas.

\section{AVALIAÇÃO DO EIXO HIPOTÁLAMO-HIPÓFISE- ADRENAL NO PÓS-OPERATÓRIO}

Níveis de hormônio adrenocorticotrófico no pósoperatório

A queda dos níveis de ACTH plasmático no PO tem sido sugerida como marcador de remissão da 
DC em alguns estudos. Graham e cols. (43) identificaram queda de $40 \%$ nos níveis de ACTH plasmático uma hora após a CTE em $82 \%$ dos indivíduos considerados em remissão para DC e uma queda menor que $40 \%$ nos níveis de ACTH plasmático no PO em $71 \%$ dos indivíduos que não obtiveram remissão da doença, não sendo, portanto, uma medida acurada para predizer a completa ressecção tumoral na DC. No estudo de Pereira e cols. (44), um valor mais baixo do ACTH plasmático no PO foi bom marcador de remissão, mas não de recidiva da DC. Acebes e cols. (4) encontraram no ponto de corte de $34 \mathrm{pg} /$ dL do ACTH plasmático no PO comum S: $80 \%$ e E: 97,5\% para identificar pacientes com remissão da DC. Para Invitti e cols. (9), houve associação entre risco de recorrência e o valor de ACTH plasmático mais elevado no PO.

\section{Teste de supressão com dexametasona}

O uso da supressão com dexametasona "overnight" foi avaliado por Bochicchio e cols. (25). Dos 510 pacientes com teste de supressão normal, 65 (12,7\%) sofreram recorrência da DC. Na publicação de Chen e cols. (37), 93\% dos pacientes com supressão do cortisol matinal das $8 \mathrm{~h}<3 \mu \mathrm{g} / \mathrm{dL}$, avaliado no terceiro dia de $\mathrm{PO}$, permaneceram em remissão da DC durante cinco anos de seguimento.

Teste estímulo com hormônio liberador de corticotrofina (CRH) ou com desmopressina (DDAVP) no pós-operatório

A resposta do cortisol/ACTH ao $\mathrm{CRH}$ exógeno logo após a cirurgia é sugerida como sendo um bom marcador de persistência do tumor hipofisário $(9,45)$. Contudo, em decorrência da dificuldade de importação do CRH humano e do seu alto custo, o uso do teste do DDAVP (desmopressina) no PO vem sendo sugerido como alternativa como indicador de persistência e recidiva da DC (41,46-48), com raros e leves efeitos adversos após sua aplicação, como náuseas e rubor facial. Os indivíduos saudáveis não costumam responder ao DDAVP (48). Assim, para os pacientes com DC responsivos ao DDAVP na avaliação pré-operatória, o desaparecimento da resposta do cortisol e/ou do ACTH ao DDAVP no PO foi sugerido como marcador de remissão da DC (47) e o retorno dessa resposta como sendo um indicador de recidiva $(41,48)$.
Estudos recentes avaliaram o uso do estímulo com CRH ou DDAVP após supressão com dexametasona para predizer remissão em longo prazo no PO da CTE dos pacientes com DC. O objetivo da dexametasona seria a supressão dos corticotrofos normais, permitindo que apenas as células tumorais respondessem ao CRH ou ao DDAVP. Salem e cols. (49) não encontraram uma boa acurácia do teste com CRH para excluir a recorrência da DC (E de $47 \%)$. Entretanto, com relação ao teste com DDAVP, Castinetti e cols. (50) acompanharam 38 pacientes submetidos à CTE por aproximadamente 60 meses e o teste alcançou S: 100\%, E: 89\% como marcador de recorrência da $\mathrm{DC}$, sinalizando a recidiva da DC com 6-60 meses de antecedência em relação aos marcadores clássicos do hipercortisolismo.

\section{Dosagem do cortisol pós-operatório}

A avaliação do cortisol no PO da CTE tem sido sugerida como a maneira mais fidedigna de predizer remissão da DC. Os protocolos de aferição variam entre os diferentes centros, seja com relação ao momento de aferição do cortisol: mais precoce (desde o PO imediato até duas semanas após a cirurgia) $(4,26,28,30,32,33,36,39,42,44,51-55)$ e/ou mais tardio (entre 1 e 6 meses de PO) $(9,30,42,44)$, seja pela utilização de diferentes esquemas de corticoide exógeno como rotina desde o período perioperatório ou apenas em caso de evidência clínica ou bioquímica de insuficiência adrenal $(4,36,52,55)$. Essa variabilidade entre os diferentes estudos e centros com relação aos protocolos de aferição do cortisol sérico no PO acarreta dificuldades na comparação dos resultados.

Na década de 1990, após a publicação em 1993 de Trainer e cols. (32) reforçada por McCance e cols. (51), acreditava-se que valores de cortisol indetectáveis (cortisol sérico $<1,8 \mu \mathrm{g} / \mathrm{dL}$ ) no $\mathrm{PO}$ eram indispensáveis para definir a remissão da DC e que níveis detectáveis de cortisol nesse período seriam marcadores de falência cirúrgica. Propunha-se, inclusive, a indicação de reintervenção cirúrgica $(31,32)$. A partir do fim da década de 1990, alguns estudos começaram a identificar casos de pacientes com níveis indetectáveis de cortisol no PO que recidivaram a DC $(25,32,43)$.

O questionamento sobre a necessidade de valores indetectáveis de cortisol no PO para definir remis- 
são e recorrência da DC iniciou após a publicação de Bochicchio e cols. (25) em 1995, na qual 33/135 $(24,4 \%)$ dos pacientes com níveis detectáveis de cortisol matinal no PO apresentaram recorrência, e 4/94 (4\%) dos pacientes com níveis indetectáveis de cortisol apresentaram recorrência. Yap e cols. (43) observaram que $7 / 61(11,5 \%)$ dos pacientes com níveis de cortisol sérico $<1,8 \mu \mathrm{g} / \mathrm{dL}$ sofreram recorrência da doença em 36 meses de seguimento. Outro estudo identificou recorrência em longo prazo de até $20 \%$ nos pacientes com insuficiência adrenal no PO (56). Observou-se também que níveis detectáveis de cortisol no PO precoce poderiam lentamente declinar ao longo dos dias e esses pacientes permaneciam em remissão por longos períodos $(36,44,57)$.

A premissa da necessidade de valores indetectáveis de cortisol no PO vem da lógica de que a supressão crônica dos corticotrofos não tumorais pelo excesso de cortisol na DC se manifestaria por níveis indetectáveis de ACTH e consequentemente de cortisol, após a remoção completa do adenoma produtor de ACTH (56). Nesse sentido, a persistência de níveis de cortisol sérico detectáveis no PO poderia ser um marcador de persistência da lesão tumoral $(34,57)$. No entanto, é possível que a adrenal hiperplásica resultante do estímulo crônico de ACTH pelo tumor hipofisário desenvolva uma semiautonomia e persista produzindo níveis detectáveis de cortisol, mesmo na ausência do estímulo tumoral do ACTH, ocasionando queda mais tardia nos níveis séricos do cortisol no PO (44). Outra especulação para esse declínio mais lento do cortisol seria a de que uma porção das células tumorais sofreria necrose após a cirurgia, com redução gradual dos níveis séricos de cortisol ao longo dos dias de PO. A menor confirmação histológica de adenoma nesses pacientes com queda mais lenta dos níveis do cortisol no PO, sugerindo necrose "in situ”, reforçaria essa teoria (57). Sugere-se, ainda, que pacientes com DC mais leve teriam menor inibição dos corticotrofos saudáveis, e a redução do cortisol $\mathrm{PO}$ poderia ser mais gradual $(52,55)$. Além disso, Pereira e cols. (44) observaram que indivíduos com DC por macroadenoma que atingiram remissão com a CTE apresentaram queda mais tardia dos níveis de cortisol no PO.

A avaliação no PO imediato por meio das dosagens de cortisol sérico mostra-se de extrema importância a curto e longo prazo na avaliação da remissão da DC e, em alguns estudos, pôde prever o risco de recidiva tumoral $(25,32,36,38,51)$. No entanto,
Pereira e cols. (44) e Chee e cols. (30) não encontraram relação dos níveis de cortisol no $\mathrm{PO}$ e risco de recidiva na DC.

Recente metanálise (58) sobre os níveis de cortisol no PO na DC incluiu 14 estudos, totalizando 786 indivíduos com níveis subnormais de cortisol no PO e 319 indivíduos com valores normais, com um seguimento variável de 2 a 9,6 anos. Essa metanálise identificou uma taxa de recorrência cumulativa da DC de 9\% (IC 95\%: 6\%-12\%) no grupo com cortisol indetectável e de 24\% (IC 95\%: 17-31\%) no grupo com cortisol normal. $\mathrm{O}$ risco relativo cumulativo de recidiva foi de 0,39 nos indivíduos com cortisol subnormal (IC 95\%: 0,25-0,47 p < 0, 001).

Muitos centros médicos administram corticoide exógeno antes, durante e após a CTE, mediante diferentes protocolos de emprego da medicação. Tais procedimentos são justificados pelo temor da potencial insuficiência adrenal central no PO (29). Contudo, por definição, os pacientes com DC possuem um eixo HHA altamente ativado e estudos de medida intraoperatória dos níveis de cortisol e ACTH observaram que tanto o ACTH quanto o cortisol elevados declinam gradualmente após a adenomectomia hipofisária completa (43). De fato, considerando a meia-vida do cortisol sérico e do ACTH, os valores de ambos ainda são detectáveis nas primeiras 12 a 14 horas de PO (59). Os glicocorticoides levam em torno de 15 minutos para inibir a transcrição do gene da POMC no corticotrofo normal (60). Além disso, mesmo em condições patológicas como na DC, aproximadamente $2 \%$ dos pacientes suprimem completamente o cortisol após teste de $2 \mathrm{mg}$ de dexametasona, indicando que há relativa sensibilidade dos corticotrofos (60). Dessa maneira, no período PO, a síntese e liberação do ACTH pelos corticotrofos tumorais remanescentes pode ser inibida pela administração de glicocorticoide exógeno, mesmo em baixas doses, especialmente por períodos mais prolongados (dias a semanas) (60). Além disso, o uso rotineiro de dexametasona pode estender ainda mais o período de supressão do eixo HHA.

É importante salientarmos a pobreza de informações a respeito do uso de medicação inibidora da esteroidogênese adrenal no período pré-operatório. Tendo em vista a meia-vida prolongada dessas medicações, podendo chegar a meses no caso do Mitotano, poucos grupos $(9,44,52)$ fazem menção ao uso ou não dessas medicações e o período de suspensão 
no pré-operatório antes da avaliação do eixo hipotálamo-hipófise-adrenal.

Portanto, o protocolo ideal de avaliação no PO da CTE seria aquele que, além do controle para o uso de medicações que interfiram no eixo HHA no pré-operatório, realizasse avaliação clínica e laboratorial do paciente no $\mathrm{PO}$, com coleta de cortisol sérico a cada 6 horas do PO e com a administração de corticoide exógeno apenas no paciente que apresentasse sintomas de insuficiência adrenal como hipotensão arterial ou valor de cortisol sérico abaixo de $2-5 \mu \mathrm{g} / \mathrm{dL}$, mesmo na ausência de sinais e sintomas clínicos de insuficiência adrenal $(36,59)$. Poucos grupos têm analisado o cortisol sérico no PO sem a reposição de glicocorticoides. Temos conhecimento de quatro publicações desses grupos $(4,36,52,55)$ descritos na tabela 3 .

Simmons e cols. (55) foram os pioneiros na avaliação da dinâmica do cortisol no PO da CTE sem uso de corticoide exógeno como rotina. Foram analisados 27 pacientes com DC por meio da medida do cortisol da meia-noite no pré-operatório e a cada 6 horas $(6 \mathrm{~h}, 12 \mathrm{~h}, 18 \mathrm{~h}$ e meia-noite) nos primeiros três dias de PO. Durante o primeiro dia de PO, identificaram excelente acurácia para um nadir de cortisol sérico abaixo de $10 \mu \mathrm{g} / \mathrm{dL}$ associado a um nadir do cortisol PO abaixo do valor do cortisol medido à meia-noite do pré-operatório, com $\mathrm{S}$ de $95,4 \% \mathrm{e} \mathrm{E}$ de $100 \%$ para remissão da DC, durante seguimento de 27 meses. No entanto, avaliando isoladamente um valor do cortisol abaixo de $10 \mu \mathrm{g} / \mathrm{dL}$ nas primeiras $24 \mathrm{~h}$ de $\mathrm{PO}$, encontrou-se $\mathrm{S}$ de $72,7 \%$ para remissão da DC. A publicação evidenciou, de forma pioneira, a segurança do uso do corticoide exógeno nesse período apenas se evidência clínica ou bioquímica de hipocortisolismo.

Rollin e cols. (36) avaliaram prospectivamente 41 pacientes, e a dosagem do cortisol sérico $<7,5 \mu \mathrm{g} / \mathrm{dL}$
10-12 dias após a CTE foi considerada um excelente marcador de remissão da doença, com S de $100 \%$ e E de $100 \%$. Entretanto, os 17 pacientes iniciais foram analisados com o uso de corticoide como rotina no transoperatório, tendo em vista a prática aceita na época. Assim, a medida do cortisol nesses pacientes foi realizada apenas uma semana após a realização da CTE. Vinte e seis indivíduos foram analisados pela medida do cortisol sérico a cada 6 h por 24 h de PO, usando corticoide apenas se necessário (sintomas de insuficiência adrenal e ou cortisol sérico abaixo de $5 \mu \mathrm{g} / \mathrm{dL}$ ), e apresentaram em $24 \mathrm{~h}$ um valor bem acurado para o nadir do cortisol: < $10 \mu \mathrm{g} / \mathrm{dL}$ para identificar a remissão da DC, com S de $90 \%$ e E de $100 \%$.

Esposito e cols. (52) estudaram prospectivamente 40 pacientes com DC submetidos à CTE com uso de corticoide exógeno apenas se insuficiência adrenal no PO, por meio da medida do cortisol das 8 horas da manhã no primeiro e no segundo dias de PO. Utilizaram como ponto de corte o nadir do cortisol $\leq 5 \mu \mathrm{g} / \mathrm{dL}$ entre essas duas medidas e identificaram 97\% indivíduos em remissão durante seguimento médio de 32 meses. A média do nadir do cortisol no PO foi de 2,05 $\pm 1,2 \mu \mathrm{g} / \mathrm{dL}$ nos indivíduos em remissão da DC e $22 \pm 12,8 \mu \mathrm{g} / \mathrm{dL}$ nos indivíduos com persistência da doença.

Acebes e cols. (4) avaliaram prospectivamente 44 indivíduos com DC operados por CTE mediante dosagem do cortisol às 8 horas da manhã do primeiro dia de PO, e o ponto de corte de $21 \mu \mathrm{g} / \mathrm{dL}$ apresentou S: $97 \%$ e E: $100 \%$ para definir remissão da DC ao longo do seguimento médio de 49 meses.

Conforme supracitado, esses quatro grupos $(4,36,52,55)$ apresentaram resultados animadores, porém com número reduzido de pacientes e tempo relativamente curto de seguimento.

Tabela 3. Avaliação do cortisol sérico sem uso de glicocorticoide no transoperatório de cirurgia hipofisária para tratamento da doença de Cushing

\begin{tabular}{lccccc}
\hline Autor, ano & $\mathbf{n}$ & Cortisol P0 & $\begin{array}{c}\text { Critério de remissão: } \\
\text { Cortisol ( } \mathbf{\mu g} / \mathbf{d L})\end{array}$ & S/E (\%) & $\begin{array}{c}\text { Seguimento } \\
\text { (meses) }\end{array}$ \\
\hline Simmons e cols., 2001 (55) & 27 & $6 / 6 \mathrm{~h}, 1^{\circ} \mathrm{dia}$ & $10 \mathrm{e}<24 \mathrm{~h}$ & $95 / 100$ & 27 \\
Rollin e cols., 2004 (36) & 26 & $6 / 6 \mathrm{~h}, 1^{\circ} \mathrm{dia}$ & 10 & $90 / 100$ & 56 \\
Esposito e cols., 2006 (52) & 40 & $8 \mathrm{~h}, 1^{\circ}, 2^{\circ} \mathrm{dia}$ & 5 & $97 / 97$ & 32 \\
Acebes e cols., 2007 (4) & 44 & $8 \mathrm{~h}, 1^{\circ} \mathrm{dia}$ & 21 & $97 / 90$ & 49 \\
\hline
\end{tabular}

n: número de pacientes; PO: pós-operatório; S: sensibilidade; E: especificidade. 24h = cortisol da meia-noite no pré-operatório. 


\section{CONCLUSÕES}

A avaliação do eixo hipotálamo-hipófise-adrenal constitui-se em importante ferramenta no arsenal diagnóstico e na avaliação da atividade da DC. Esse tema torna-se mais desafiador à medida que cada vez mais pacientes com doença mais leve procuram auxílio médico. Após o tratamento, a melhor maneira de prever a remissão e a recorrência da DC em longo prazo permanece em discussão. A despeito da raridade de estudos analisando o cortisol sérico após a CTE nos pacientes com DC sem uso de corticoide $(4,36,52,55)$, do pequeno número de pacientes estudados, dos diferentes pontos de corte discriminatórios para o cortisol e dos diferentes momentos de mensuração do cortisol, o cortisol sérico no PO constitui-se em importante auxiliar no diagnóstico de remissão e no manejo pós-operatório dos pacientes com DC.

Declaração: os autores declaram não haver conflitos de interesse científico neste estudo.

\section{REFERÊNCIAS}

1. Nieman LK, Biller BM, Findling JW, Newell-Price J, Savage MO, Stewart PM, et al.The diagnosis of Cushing's syndrome: an Endocrine Society Clinical Practice Guideline. J Clin Endocrinol Metab. 2008;93:1526-40.

2. Clayton RN, Raskauskiene D, Reulen RC, Jones PW. Mortality and morbidity in Cushing's disease over 50 years in Stoke-on-Trent, UK: audit and meta-analysis of literature. J Clin Endocrinol Metab. 2011;96:632-42.

3. Newell-Price J, Trainer P, Besser M, Grossman A. The diagnosis and differential diagnosis of Cushing's syndrome and pseudo-Cushing's states. Endocr Rev. 1998;19:647-72.

4. Acebes JJ, Martino J, Masuet C, Montanya E, Soler J. Early post-operative ACTH and cortisol as predictors of remission in Cushing's disease. Acta Neurochir (Wien). 2007;149:471-7.

5. Chanson P, Salenave S. Metabolic syndrome in Cushing's syndrome. Neuroendocrinology. 2010;92:96-101.

6. Indicadores de fatores de risco e de proteção. Tx prevalência diabetes melito segundo região (capitais) período: 2008. Disponível em: <www.datasus.gov.br>. Acesso em: 23 Ago 2011.

7. Indicadores de fatores de risco e de proteção. Tx prevalência hipertensão arterial segundo região (capitais) período: 2008. Disponível em: <www.datasus.gov.br>. Acesso em: 23 Ago 2011.

8. Chrousos GP. Glucocorticoid therapy and Cushing's syndrome (atualizado em 11 março de 2009). Disponível em: <emedicine. medscape.com/article/921086-overview>. Acesso em: 23 Ago 2011.

9. Invitti C, Pecori Giraldi F, de Martin M, Cavagnini F. Diagnosis and management of Cushing's syndrome: results of an Italian multicentre study. Study Group of the Italian Society of Endocrinology on the Pathophysiology of the Hypothalamic-Pituitary-Adrenal Axis. J Clin Endocrinol Metab. 1999;84:440-8.

10. Wood PJ, Barth JH, Freedman DB, Perry L, Sheridan B. Evidence for the low dose dexamethasone suppression test to screen for
Cushing's syndrome--recommendations for a protocol for biochemistry laboratories. Ann Clin Biochem. 1997;34:222-9.

11. Lin CL, Wu TJ, Machacek DA, Jiang NS, Kao PC. Urinary free cortisol and cortisone determined by high performance liquid chromatography in the diagnosis of Cushing's syndrome. J Clin Endocrinol Metab. 1997;82:151-5.

12. Martinelli CE Jr, Sader SL, Oliveira EB, Daneluzzi JC, Moreira AC. Salivary cortisol for screening of Cushing's syndrome in children. Clin Endocrinol (Oxf). 1999;51:67-71.

13. Newell-Price J, Trainer P, Perry L, Wass J, Grossman A, Besser M. A single sleeping midnight cortisol has $100 \%$ sensitivity for the diagnosis of Cushing's syndrome. Clin Endocrinol (Oxf). 1995;43:545-50.

14. Papanicolaou DA, Yanovski JA, Cutler GB Jr, Chrousos GP, Nieman LK. A single midnight serum cortisol measurement distinguishes Cushing's syndrome from pseudo-Cushing states. J Clin Endocrinol Metab. 1998;83:1163-7.

15. Friedman TC, Ghods DE, Shahinian HK, Zachery L, Shayesteh $\mathrm{N}$, Seasholtz $\mathrm{S}$, et al. High prevalence of normal tests assessing hypercortisolism in subjects with mild and episodic Cushing's syndrome suggests that the paradigm for diagnosis and exclusion of Cushing's syndrome requires multiple testing. Horm Metab Res. 2010;42:874-81.

16. Isidori AM, Kaltsas GA, Pozza C, Frajese V, Newell-Price J, Reznek $\mathrm{RH}$, et al. The ectopic adrenocorticotropin syndrome: clinical features, diagnosis, management, and long-term follow-up. J Clin Endocrinol Metab. 2006;91:371-7.

17. Kronenberg HM, Melmed S, Polonsky KS, Larsen RP. Williams Textbook of Endocrinology. 11nd ed. Philadelphia: Elsevier Ltda.; 2009. p. 445-503.

18. Salgado LR, Fragoso MC, Knoepfelmacher M, Machado MC, Domenice S, Pereira MA, et al. Ectopic ACTH syndrome: our experience with 25 cases. Eur J Endocrinol. 2006;155:725-33.

19. Pecori Giraldi F, Pivonello R, Ambrogio AG, De Martino MC, De Martin M, Scacchi M, et al. The dexamethasone-suppressed corticotropin-releasing hormone stimulation test and the desmopressin test to distinguish Cushing's syndrome from pseudo-Cushing's states. Clin Endocrinol (Oxf). 2007;66:251-7.

20. Arnaldi G,Tirabassi G, Papa R, Furlani G, Trementino L, Cardinaletti $M$, et al. Human corticotrophin releasing hormone test performance in the differential diagnosis between Cushing's disease and pseudo-Cushing state is enhanced by combined ACTH and cortisol analysis. Eur J Endocrinol. 2009;160:891-8.

21. de KeyzerY, René $P$, Beldjord $C$, Lenne F, Bertagna X. Overexpression of vasopressin (V3) and corticotrophin-releasing hormone receptor genes in corticotroph tumours. Clin Endocrinol (Oxf). 1998;49:475-82.

22. Moro $M$, Putignano $P$, Losa $M$, Invitti $C$, Maraschini $C$, Cavagnini $\mathrm{F}$. The desmopressin test in the differential diagnosis between Cushing's disease and pseudo-Cushing states. J Clin Endocrinol Metab. 2000;10:3569-74.

23. Tirabassi G, Faloia E, Papa R, Furlani G, Boscaro M, Arnaldi G. Use of the desmopressin test in the differential diagnosis of pseudo-Cushing state from Cushing's disease. J Clin Endocrinol Metab. 2010;95:1115-22.

24. Mancini T, Porcelli T, Giustina A. Treatment of Cushing's disease: overview and recent findings. Ther Clin Risk Manag. 2010;6:505-16.

25. Bochicchio D, Losa M, Buchfelder M. Factors influencing the immediate and late outcome of Cushing's disease treated by transsphenoidal surgery: a retrospective study by the European Cushing's Disease Survey Group. J Clin Endocrinol Metab. 1995;80:3114-20.

26. Hammer GD, Tyrrell JB, Lamborn KR, Applebury CB, Hannegan ET, Bell S, et al. Transsphenoidal microsurgery for Cushing's di- 
sease: initial outcome and long-term results. J Clin Endocrinol Metab. 2004;89:6348-57.

27. Sonino N, Zielezny M, Fava GA, Fallo F, Boscaro M. Risk factors and long-term outcome in pituitary-dependent Cushing's disease. J Clin Endocrinol Metab. 1996;81:2647-52.

28. Rollin G, Ferreira NP, Czepielewski MA. Prospective evaluation of transsphenoidal pituitary surgery in 108 patients with Cushing's disease. Arq Bras Endocrinol Metabol. 2007;51:1355-61.

29. Blevins LS Jr, Christy JH, Khajavi M, Tindall GT. Outcomes of therapy for Cushing's disease due to adrenocorticotropin secreting pituitary macroadenomas. J Clin Endocrinol Metab. 1998;83:63-7.

30. Chee GH, Mathias DB, James RA, Kendall-Taylor P. Transsphenoidal pituitary surgery in Cushing's disease: can we predict outcome? Clin Endocrinol (Oxf). 2001;54:617-26.

31. Locatelli M, Vance ML, Laws ER. Clinical review: the strategy of immediate reoperation for transsphenoidal surgery for Cushing's disease. J Clin Endocrinol Metab. 2005;90:5478-82.

32. Trainer PJ, Lawrie HS, Verhelst J, Howlett TA, Lowe DG, Grossman $A B$, et al. Transsphenoidal resection in Cushing's disease: undetectable serum cortisol as the definition of successful treatment. Clin Endocrinol (Oxf). 1993;38:73-8.

33. Estrada J, García-Uría J, Lamas C, Alfaro J, Lucas T, Diez S, et al. The complete normalization of the adrenocortical function as the criterion of cure after transsphenoidal surgery for Cushing's disease. J Clin Endocrinol Metab. 2001;86:5695-9.

34. Czepielewski MA, Rollin GA, Casagrande A, Ferreira NP. Criteria of cure and remission in Cushing's disease: an update. Arq Bras Endocrinol Metabol. 2007;51:1362-72.

35. Burke CW, Adams CB, Esiri MM, Morris C, Bevan JS. Transsphenoidal surgery for Cushing's disease: does what is removed determine the endocrine outcome? Clin Endocrinol (Oxf). 1990;33:525-37.

36. Rollin GAF, Ferreira NP, Junges M, Gross JL, Czepielewski MA. Patients with Cushing's disease dynamics of serum cortisol levels after transsphenoidal surgery in a cohort of patients with Cushing's disease. J Clin Endocrinol Metab. 2004;89:1131-9.

37. Chen JC, Amar AP, Choi S, Singer $P$, Couldwell WT, Weiss MH. Transsphenoidal microsurgical treatment of Cushing disease: postoperative assessment of surgical efficacy by application of an overnight low-dose dexamethasone suppression test. J Neurosurg. 2003;98:967-73.

38. Guilhaume B, Bertagna $X$, Thomsen M, Bricaire C, Vila-Porcile E, Olivier $L$, et al. Transsphenoidal pituitary surgery for the treatment of Cushing's disease: results in 64 patients and long term follow-up studies. J Clin Endocrinol Metab. 1988;66:1056-64.

39. Atkinson AB, Kennedy A, Wiggam MI, McCance DR, Sheridan B. Long-term remission rates after pituitary surgery for Cushing's disease: the need for long-term surveillance. Clin Endocrinol (Oxf). 2005;63:549-59.

40. George DH, Scheithauer BW, Kovacs K, Horvath E, Young WF $\mathrm{Jr}$, Lloyd RV, et al. Crooke's cell adenoma of the pituitary: an aggressive variant of corticotroph adenoma. Am J Surg Pathol. 2003;27:1330-6.

41. Devoe DJ, Miller WL, Conte FA, Kaplan SL, Grumbach MM, Rosenthal SM, et al. Long-term outcome in children and adolescents after transsphenoidal surgery for Cushing's disease. Clin Endocrinol Metab. 1997;82:3196-202.

42. Yap LB, Turner HE, Adams CB, Wass JA. Undetectable postoperative cortisol does not always predict long-term remission in Cushing's disease: a single centre audit. Clin Endocrinol (Oxf). 2002;56:25-31.

43. Graham KE, Samuels MH, Raff H, Barnwell SL, Cook DM. Intraoperative adrenocorticotropin levels during transsphenoidal surgery for Cushing's disease do not predict cure. J Clin Endocrinol Metab. 1997;82:1776-9.
44. Pereira AM, van Aken MO, van Dulken H, Schutte PJ, Biermasz NR, Smit JW, et al. Long-term predictive value of postsurgical cortisol concentrations for cure and risk of recurrence in Cushing's disease. J Clin Endocrinol Metab. 2003;88:5858-64.

45. Vignati F, Berselli ME, Loi P. Early postoperative evaluation in patients with Cushing's disease: usefulness of ovine corticotrophin releasing hormone test in the prediction of recurrence of disease. Eur J Endocrinol. 1994;130:235-41.

46. Dall'Asta C, Barbetta L, Bonavina L, Beck-Peccoz P, Ambrosi B. Recurrence of Cushing's disease preceded by the reappearance of ACTH and cortisol responses to desmopressin test. Pituitary. 2004;7:183-8.

47. Losa M, Mortini P, Dylgjeri S, Barzaghi R, Franzin A, Mandelli C, et al. Desmopressin stimulation test before and after pituitary surgery in patients with Cushing's disease. Clin Endocrinol (Oxf). 2001;55:61-8.

48. Romanholi DJ, Machado MC, Pereira CC, Danilovic DS, Pereira MA, Cescato VA, et al. Role for postoperative cortisol response to desmopressin in predicting the risk for recurrent Cushing's disease. Clin Endocrinol (Oxf). 2008;69:117-22.

49. Salem V, Dhillo WS, Meeran K, Donaldson M, Martin NM. Dexamethasone-suppressed corticotrophin-releasing hormone-stimulation test does not reliably diagnose or predict recurrence of Cushing disease. Clin Chem. 2010;56:1031-4.

50. Castinetti F, Martinie M, Morange I, Dufour H, Sturm N, Passagia JG, et al. A combined dexamethasone desmopressin test as an early marker of postsurgical recurrence in Cushing's disease. J Clin Endocrinol Metab. 2009;94:1897-903.

51. McCance DR, Gordon DS, Fanin TT, Hadden DR, Kennedy L, Sheridan $B$, et al. Assessment of endocrine function after transsphenoidal surgery for Cushing's disease. Clin Endocrinol (Oxf). 1993;38:79-86.

52. Esposito F, Dusick JR, Cohan P, Moftakhar P, McArthur D, Wang C, et al. Clinical review: early morning cortisol levels as a predictor of remission after transsphenoidal surgery for Cushing's disease. J Clin Endocrinol Metab. 2006;91:7-13.

53. Lindsay JR, Oldfield EH, Stratakis CA, Nieman LK. The postoperative basal cortisol and $\mathrm{CRH}$ tests for prediction of long-term remission from Cushing's disease after transsphenoidal surgery. $\mathrm{J}$ Clin Endocrinol Metab. 2011;96:2057-64.

54. Alwani RA, de Herder WW, van Aken MO, van den Berge JH, Delwel EJ, Dallenga AH, et al. Biochemical predictors of outcome of pituitary surgery for Cushing's disease. Neuroendocrinology. 2010;91:169-78.

55. Simmons NE, Alden TD, Thorner MO, Laws ER Jr. Serum cortisol response to transsphenoidal surgery for Cushing disease. J Neurosurg. 2001;95:1-8.

56. Fitzgerald PA, Aron DC, Findling JW, Brooks RM, Wilson CB, Forsham $\mathrm{PH}$, et al. Cushing's disease: transient secondary adrenal insufficiency after selective removal of pituitary microadenomas; evidence for a pituitary origin. J Clin Endocrinol Metab. 1982;54:413-22.

57. Valassi E, Biller BM, Swearingen B, Pecori Giraldi F, Losa M, Mortini $P$, et al. Delayed remission after transsphenoidal surgery in patients with Cushing's disease. J Clin Endocrinol Metab. 2010;95:601-10.

58. Sughrue ME, Shah JK, Devin JK, Kunwar S, Blevins LS Jr. Utility of the immediate postoperative cortisol concentrations in patients with Cushing's disease. Neurosurgery. 2010;67:688-95.

59. AbdelMannan D, Selman WR, Arafah BM. Peri-operative management of Cushing's disease. Rev Endocr Metab Disord. 2010;11:127-34.

60. Newell-Price J. Transsphenoidal surgery for Cushing's disease: defining cure and following outcome. Clin Endocrinol (Oxf). 2002;56:19-21. 


\section{Correção do Artigo}

\section{Avaliação do eixo hipotálamo-hipófise adrenal no diagnóstico e na remissão da doença de Cushing}

Fabíola Costenaro, Ticiana C. Rodrigues, Guilherme A. F. Rollin, Mauro A. Czepielewski

Arq Bras Endocrinol Metab. 2012;56(3):159-67

Na Tabela 1, onde se lê:

Tabela 1. Performance dos testes no diagnóstico da síndrome de Cushing

\begin{tabular}{lccc}
\hline \multicolumn{1}{c}{ Teste } & Ponto de corte & Sensibilidade (\%) & Especificidade (\%) \\
\hline Cortisol após 1 mg de dexametasona "overnight" $(3,9,10)$ & $\geq 1,8 \mu \mathrm{g} / \mathrm{dL}$ & 95 & 80 \\
& $\geq 5 \mu \mathrm{g} / \mathrm{dL}$ & 85 & 95 \\
Cortisolúria de 24h (3,11) & $>$ Limite superior da normalidade & $90-100$ & $50-98$ \\
Cortisol salivar à meia-noite (12) & $>145 \mathrm{ng} / \mathrm{dL}$ & $95-100$ & $93-100$ \\
Cortisol sérico à meia-noite (13,14) & Dormindo: $>1,8 \mu \mathrm{g} / \mathrm{dL}$ & 100 & 20 \\
& Acordado: $>7,5 \mu \mathrm{g} / \mathrm{dL}$ & 96 & $83-96$ \\
Teste de Liddle II (1,3) & $>1,8-5 \mu \mathrm{g} / \mathrm{dL}$ & $67-95$ & $70-100$ \\
\hline
\end{tabular}

CRH: hormônio liberador de cortrosina; DDAVP: desmopressina.

Leia-se:

Tabela 1. Performance dos testes no diagnóstico da síndrome de Cushing

\begin{tabular}{lccc}
\hline \multicolumn{1}{c}{ Teste } & Ponto de corte & Sensibilidade (\%) & Especificidade (\%) \\
\hline Cortisol após 1 mg de dexametasona "overnight" (3,9,10) & $\geq 1,8 \mu \mathrm{g} / \mathrm{dL}$ & 95 & 80 \\
& $\geq 5 \mu \mathrm{g} / \mathrm{dL}$ & 85 & 95 \\
Cortisolúria de 24h (3,11) & $>$ Limite superior da normalidade & $90-100$ & $50-98$ \\
Cortisol salivar à meia-noite (12) & $>145 \mathrm{ng} / \mathrm{dL}$ & $95-100$ & $93-100$ \\
Cortisol sérico à meia-noite (13,14) & Dormindo: $>1,8 \mu \mathrm{g} / \mathrm{dL}$ & 100 & 20 \\
& Acordado: $>7,5 \mu \mathrm{g} / \mathrm{dL}$ & 96 & $83-96$ \\
Teste de Liddle I $(1,3)$ & $>1,8-5 \mu \mathrm{g} / \mathrm{dL}$ & $67-95$ & $70-100$ \\
\hline
\end{tabular}

CRH: hormônio liberador de cortrosina; DDAVP: desmopressina. 